\title{
Determinação da condutividade hidráulica e da sorvidade de um solo com infiltrômetro a disco
}

\author{
Antonio C. D. Antonino', Rafael Angulo-Jaramillo², Eduardo S. de Souza1', André Maciel Netto', \\ Clemente J. G. Carneiro ${ }^{1} \&$ Abelardo A. A. Montenegro ${ }^{3}$
}

\begin{abstract}
${ }^{1}$ UFPE. Av. Prof. Luís Freire 1000, CEP 50740-540, Recife, PE. Fone: (81) 3271-8252. E-mail: acda@npd.ufpe.br (Foto) 2 LTHE (UMR 5564 CNRS, INPG,IRD,UJF), BP 53, 38041 Grenoble cedex 9, França. E-mail: angulo@hmg.inpg.fr 3 UFRPE. Dois Irmãos, CEP 50670-910, Recife, PE. E-mail: monte@hotlink.com.br
\end{abstract}

Protocolo $097-14 / 08 / 2000$

\begin{abstract}
Resumo: Determinou-se a condutividade hidráulica e a sorvidade, a partir de dados de infiltração acumulada, utilizando-se um infiltrômetro a disco, em duas camadas de um Neossolo Flúvico (uma superficial, $15-25 \mathrm{~cm}$ de profundidade, argilosa, e outra $50-70 \mathrm{~cm}$ de profundidade, arenosa) da vazante do açude Flocos, localizado no município de Tuparetama, PE. Para a caracterização hidrodinâmica, os ensaios de infiltração foram realizados aplicando-se potenciais de fornecimento de água de $0,-3,-6$ e $-12 \mathrm{~cm}$ de coluna de água, utilizando um infiltrômetro a disco, com base de $80 \mathrm{~mm}$ de diâmetro. Três métodos foram empregados para se determinar a condutividade hidráulica (K) e a sorvidade (S) baseados no conhecimento prévio da curva de retenção da água no solo, obtida pelo ajuste das funcionais propostas por: i) van Genuchten (VG); ii) Russo (GR); e iii) Zhang \& van Genuchten (ZV) aos valores experimentais de campo e de laboratório. A equação da infiltração acumulada em função do tempo descreveu com precisão os ensaios de infiltração realizados, apresentando coeficientes de determinação $\left(R^{2}\right)$ superiores a 0,995. O método GR parece ser o menos recomendado, pois superestima sistematicamente e prediz satisfatoriamente a condutividade hidráulica normalizada para ambas as camadas, enquanto o método VG a prediz razoavelmente, para ambas as camadas.
\end{abstract}

Palavras-chave: condutividade hidráulica, sorvidade, infiltrômetro a disco

\section{Determination of hydraulic conductivity and sorptivity of soil using a disk infiltrometer}

\begin{abstract}
Hydraulic conductivity (K) and sorptivity (S) of two layers with different textural classes of a Fluvents soil from Flocos dams, located in Tuparetama, PE, Brazil were determined using the cumulative infiltration data measured with an infiltrometer disk. During the infiltration experiments, water was applied to the soil by an infiltrometer disk at potentials equivalent to $0,-3,-6$ e -12 $\mathrm{cm}$ of water columm. Three methods were used to evaluate $\mathrm{K}$ and S: i) van Genuchten (VG); ii) Russo (GR); e iii) Zhang \& van Genuchten (ZV). These methods based on the previous knowledge of the soil-moisture characteristic curve, which were obtained by the adjustment of the functions proposed by each method. The equation of cumulative infiltration described with precision the infiltration experiments, and yielded determination coefficients $\left(R^{2}\right)$ greater than 0.995 . The method GR is the less adequate, since it systematically overestimated values and did not predict well the normalized $\mathrm{K}$ for the two soil layers. On the other hand, the VG method yielded good predictions of normalized $\mathrm{K}$ for both soil layers.
\end{abstract}

Key words: hydraulic conductivity, sorptivity, and disk infiltrometer

\section{INTRODUÇÃO}

As propriedades hidráulicas da zona não saturada controlam os fluxos de água no solo e influenciam fortemente a redistribuição da precipitação pluviométrica e muitos processos geomorfológicos, geoquímicos ou ecológicos. As propriedades hidráulicas do solo são parâmetros fundamentais para se compreender e modelar os processos de infiltração, evapotranspiração e transporte de solutos (Angulo-Jaramillo et al., 2000).

A infiltrometria a disco (Smettem \& Clothier, 1989; Thony et al., 1991; Haverkamp et al., 1994; Angulo-Jaramillo et al., 1996; Vandervaere et al., 1997; Zhang, 1997; Borges et al., 1999) é um método desenvolvido recentemente e que permite a estimativa, no campo, da condutividade hidráulica próxima à saturação. Vários métodos têm sido desenvolvidos para se estimar as 
propriedades hidráulicas de solos a partir de medidas de fluxos transitórios ou estacionários da infiltração, obtidas com um infiltrômetro a disco ajustado a uma sucção $h_{0}$. Os métodos se baseiam em soluções quasi-analíticas da equação de fluxos em perfil de solo homogêneo ou na solução numérica da mesma equação, utilizando técnicas inversas, seja o solo homogêneo ou não (Angulo-Jaramillo et al., 2000).

Zhang (1997) propôs uma solução numérica com dois termos para descrever o processo de infiltração a partir do infiltrômetro a disco, para qualquer tempo de infiltração:

$$
\mathrm{I}=\mathrm{C}_{1} \mathrm{t}^{1 / 2}+\mathrm{C}_{2} \mathrm{t}
$$

onde $\mathrm{C}_{1}$ e $\mathrm{C}_{2}$ são parâmetros relacionados à sorvidade, $\mathrm{S}\left(\mathrm{h}_{0}\right)$, e à condutividade hidráulica, $\mathrm{K}\left(\mathrm{h}_{0}\right)$ do solo, pelas expressões:

$$
\begin{gathered}
\mathrm{C}_{1}\left(\mathrm{~h}_{0}\right)=\mathrm{A}_{1} \mathrm{~S}\left(\mathrm{~h}_{0}\right) \\
\mathrm{C}_{2}\left(\mathrm{~h}_{0}\right)=\mathrm{A}_{2} \mathrm{~K}\left(\mathrm{~h}_{0}\right)
\end{gathered}
$$

em que: $A_{1}$ e $A_{2}$ - coeficientes adimensionais e $h_{0}(\leq 0)$ - valor da pressão usada no infiltrômetro a disco durante o processo de infiltração.

Utilizando os resultados de várias simulações numéricas da infiltração tridimensional axissimétrica a partir de um infiltrômetro a disco, em diferentes tipos de solo de características hidrodinâmicas contrastadas e diversas condições iniciais e de fronteira, Zhang (1997) estabeleceu relações empíricas para os coeficientes $\mathrm{A}_{1}$ e $\mathrm{A}_{2}$ das Eqs. 2 e 3, respectivamente. Estes coeficientes foram estabelecidos para cada um dos funcionais propostos por: i) van Genuchten (1980), ii) Gardner (1958) e Russo (1998), e iii) Zhang \& van Genuchten (1994), definindo, respectivamente, os métodos VG, GR e ZV. A partir da infiltrometria a disco e do tipo de funcional escolhido, é possível utilizar-se um desses métodos, conjuntamente com a equação de infiltração (Eq. 1) para se determinar $\mathrm{S}\left(\mathrm{h}_{0}\right)$ e $\mathrm{K}\left(\mathrm{h}_{0}\right)$.

O objetivo deste trabalho foi determinar a condutividade hidráulica e a sorvidade em duas camadas, uma argilosa e a outra arenosa, de um solo neossolo flúvico, utilizando-se os três métodos propostos por Zhang (1997) a partir de experimentos de infiltração realizados com o infiltrômetro a disco.

\section{MATERIAL E MÉTODOS}

\section{Infiltrômetro a disco}

O infiltrômetro a disco com sucção controlada permite que se realizem medidas no campo para caracterizar os parâmetros fenomenológicos da infiltrabilidade, como a condutividade hidráulica e a sorvidade capilar. O infiltrômetro a disco, utilizado com uma base de $80 \mathrm{~mm}$ de diâmetro, foi concebido por Perroux \& White (1988). Um tubo graduado funciona como reservatório de alimentação para o fornecimento de água e um outro reservatório tubular, contendo um tubo de aeração que pode ser deslocado verticalmente, possibilitando uma pressão constante menor ou igual à pressão atmosférica de fornecimento de água, $\mathrm{h}_{0}$, na base do infiltrômetro. $\mathrm{O}$ funcionamento detalhado do infiltrômetro a disco encontra-se nos trabalhos de Thony et al. (1991) e Borges et al. (1999).

O infiltrômetro é colocado no solo sobre uma superfície plana e horizontal preparada previamente. A base do disco é coberta com uma tela de náilon com abertura de $20 \mu \mathrm{m}$. Uma camada de areia fina é usada para garantir bom contato hidráulico entre o disco e o solo. A leitura da variação no tempo do nível de água no reservatório de alimentação permite a obtenção da infiltração acumulada (Borges et al., 1999).

\section{Determinação de parâmetros hidráulicos (Zhang, 1997)}

As variações do nível do reservatório de alimentação do infiltrômetro durante o processo de infiltração, permite determinar a sorvidade $\mathrm{S}\left(\mathrm{h}_{0}\right)$ e a condutividade hidráulica $\mathrm{K}\left(\mathrm{h}_{0}\right)$ utilizando-se das relações dadas por:

$$
\begin{aligned}
& \mathrm{S}\left(\mathrm{h}_{0}\right)=\mathrm{C}_{1} / \mathrm{A}_{1} \\
& \mathrm{~K}\left(\mathrm{~h}_{0}\right)=\mathrm{C}_{2} / \mathrm{A}_{2}
\end{aligned}
$$

$\mathrm{C}_{1}$ e $\mathrm{C}_{2}$ são obtidos pelo ajuste da Eq. 1 aos dados de infiltração acumulada. $\mathrm{A}_{1}$ e $\mathrm{A}_{2}$ podem ser calculados pelos métodos $\mathrm{VG}$, GReZV.

\section{Método van Genuchten - VG}

O funcional tipo van Genuchten (1980) é dado por:

$$
\Theta(h)=\frac{\theta-\theta_{\mathrm{r}}}{\theta_{\mathrm{s}}-\theta_{\mathrm{r}}}=\left[1+|\alpha \mathrm{h}|^{\mathrm{n}}\right]^{-\mathrm{m}}
$$

$\operatorname{com} m=1-1 / n(n>1) e$

$$
\mathrm{K}(\Theta)=\mathrm{K}_{\mathrm{s}} \Theta^{1 / 2}\left[1-\left(1-\Theta^{1 / m}\right)^{\mathrm{m}}\right]^{2}
$$

em que: $\theta_{\mathrm{r}}, \theta_{\mathrm{s}}$, $\alpha$ e $\mathrm{K}_{\mathrm{s}}$ - representam as variáveis de estado do sistema (umidades volumétricas residual e de saturação, inverso da pressão de borbulhamento e condutividade hidráulica do solo na saturação, respectivamente), n e m - parâmetros de forma das curvas ajustadas.

Os coeficientes $A_{1}$ e $A_{2}$ se expressam como:

$$
\begin{array}{cc}
\mathrm{A}_{1}=\frac{1,4 \mathrm{~b}^{0,5}\left(\theta_{0}-\theta_{\mathrm{i}}\right)^{0,25} \exp \left[3(\mathrm{n}-1,9) \alpha \mathrm{h}_{0}\right]}{\left(\alpha \mathrm{r}_{0}\right)^{0,15}} & \\
\mathrm{~A}_{2}=\frac{11,65\left(\mathrm{n}^{0,1}-1\right) \exp \left[2,92(\mathrm{n}-1,9) \alpha \mathrm{h}_{0}\right]}{\left(\alpha \mathrm{r}_{0}\right)^{0,91}} & \mathrm{n} \geq 1,9 \\
\mathrm{~A}_{2}=\frac{11,65\left(\mathrm{n}^{0,1}-1\right) \exp \left[7,5(\mathrm{n}-1,9) \alpha \mathrm{h}_{0}\right]}{\left(\alpha \mathrm{r}_{0}\right)^{0,91}} & \mathrm{n}<1,9
\end{array}
$$


sendo que: $h_{0}(\leq 0)$ - representa a tensão aplicada pelo infiltrômetro, $\mathrm{r}_{0}$ - raio do infiltrômetro, $\theta_{0}$ - conteúdo de água em $\mathrm{h}_{0}, \theta_{\mathrm{i}}$ o conteúdo de água inicial e b um parâmetro igual a 0,55 (Warrick \& Broadbridge, 1992).

\section{Método Gardner e Russo - GR}

O funcional tipo Russo (1988) é dado por:

$$
\Theta(h)=\frac{\theta-\theta_{\mathrm{r}}}{\theta_{\mathrm{s}}-\theta_{\mathrm{r}}}=[\exp (\alpha \mathrm{h})(1-\alpha \mathrm{h} / 2)]^{2 /(\mathrm{n}+2)}
$$

e o funcional tipo Gardner (1958) por:

$$
\mathrm{K}(\mathrm{h})=\mathrm{K}_{\mathrm{s}} \exp (\alpha \mathrm{h}) \quad \mathrm{h} \leq 0
$$

Os coeficientes $A_{1}$ e $A_{2}$ se expressam como:

$$
\begin{gathered}
A_{1}=\frac{1,34 b^{0,5}\left(\theta_{0}-\theta_{i}\right)^{0,25} n^{0,115} \exp \left(0,025 \alpha h_{0}\right)}{\left(\alpha r_{0}\right)^{0,225}} \\
A_{2}=1+\frac{1,388}{\alpha r_{0}}
\end{gathered}
$$

\section{Método Zhang \& van Genuchten - ZV}

O funcional tipo Zhang \& van Genuchten (1994) é dado por:

$$
\Theta(h)=\frac{\theta-\theta_{\mathrm{r}}}{\theta_{\mathrm{s}}-\theta_{\mathrm{r}}}=\frac{1+|\alpha \mathrm{h}|}{1+|\alpha \mathrm{h}|+\mathrm{n}|\alpha \mathrm{h}|^{2}}
$$

e

$$
\mathrm{K}(\Theta)=\mathrm{K}_{\mathrm{s}} \Theta^{18 / \mathrm{n}^{0,2}}
$$

Os coeficientes $A_{1}$ e $A_{2}$ se expressam como:

$$
\begin{gathered}
\mathrm{A}_{1}=\frac{0,82 \mathrm{~b}^{0,5}\left(\theta_{0}-\theta_{\mathrm{i}}\right)^{0,1} \exp \left(1,6 \mathrm{n}^{0,224} \alpha \mathrm{h}_{0}\right)}{\mathrm{n}^{0,153}\left(\alpha \mathrm{r}_{0}\right)^{0,225}} \\
\mathrm{~A}_{2}=\frac{0,54 \exp \left(2,9 \mathrm{n}^{0,34} \alpha \mathrm{h}_{0}\right)}{\mathrm{n}^{0,39}\left(\alpha \mathrm{r}_{0}\right)^{0,91}}
\end{gathered}
$$

\section{Comparação entre métodos e modelos teóricos}

A consistência da predição do modelo teórico correspondente a cada método foi comparada utilizando-se quatro critérios estatísticos diferentes: i) o erro quadrático médio, EQM; ii) a razão de desvios, $\mathrm{RD}$; iii) a eficiência da modelagem, EM, e iv) o coeficiente de massa residual, CMR, calculados por Loague \& Green (1991):

$$
\mathrm{EQM}=\left[\frac{\sum_{\mathrm{i}=1}^{\mathrm{N}}(\mathrm{Ti}-\mathrm{Mi})^{2}}{\mathrm{~N}}\right]^{\frac{1}{2}} 100 / \overline{\mathrm{M}}
$$

$\mathrm{EM}=\left[\frac{\sum_{\mathrm{i}=1}^{\mathrm{N}}(\mathrm{Mi}-\overline{\mathrm{M}})^{2}-\sum_{\mathrm{i}=1}^{\mathrm{N}}(\mathrm{Ti}-\mathrm{Mi})^{2}}{\sum_{\mathrm{i}=1}^{\mathrm{N}}(\mathrm{Mi}-\overline{\mathrm{M}})^{2}}\right]$

$$
\mathrm{CMR}=\left[\frac{\sum_{\mathrm{i}=1}^{\mathrm{N}} \mathrm{Mi}-\sum_{\mathrm{i}=1}^{\mathrm{N}} \mathrm{Ti}}{\sum_{\mathrm{i}=1}^{\mathrm{N}} \mathrm{Mi}}\right]
$$

em que: Ti - representa os valores das condutividades hidráulicas calculadas pelos modelos teóricos (Eqs. 7, 12 e 16); Mi e $\bar{M}$ - valores experimentais e média dos valores das condutividades hidráulicas, respectivamente, e $\mathrm{N}$ é o número de determinações.

\section{Experimentos de infiltração}

As medidas para a determinação da sorvidade e da

\begin{tabular}{|c|c|c|c|c|c|}
\hline \multirow{2}{*}{$\begin{array}{c}\text { Camada } \\
\mathrm{cm}\end{array}$} & \multirow{2}{*}{$\begin{array}{c}\rho \\
\mathrm{Mg} \mathrm{m}^{-3}\end{array}$} & Areia & Silte & Argila & \multirow{2}{*}{ Classe Textura } \\
\hline & & & $\mathrm{g} \mathrm{kg}^{-1}$ & & \\
\hline $15-25$ & $1,42 \pm 0,01$ & 380 & 207 & 413 & Argilosa \\
\hline $50-70$ & $1,58 \pm 0,02$ & 902 & 25 & 73 & Arenosa \\
\hline
\end{tabular}
condutividade hidráulica foram efetuadas numa parcela solo nu, da vazante do açude Flocos, localizado no município de Tuparetama, $\mathrm{PE}\left(7^{\circ} 36^{\prime} \mathrm{S}\right.$ e $\left.37^{\circ} 18^{\prime} \mathrm{O}\right)$. O solo é caracterizado como Neossolo Flúvico (EMBRAPA, 1999) apresentando aumento de massa específica global com a profundidade e camada superficial bastante argilosa, seguida de uma camada por demais arenosa (Tabela 1).

Tabela 1. Análise granulométrica e massa específica global $(\rho)$ do solo de Flocos

As curvas de retenção da água no solo, nas profundidades de 20 e $60 \mathrm{~cm}$ (representativas das camadas de $15-25$ e $50-70 \mathrm{~cm}$, respectivamente) foram obtidas pelo ajuste dos funcionais propostos por: i) van Genuchten (1980), ii) Russo (1988) e iii) 
Zhang \& van Genuchten (1994) aos valores obtidos em campo, a partir das medidas tensiométricas e neutrônicas (empregando-se uma sonda de nêutrons) e aos valores obtidos em laboratório, determinados utilizando-se a "panela de pressão" com placa porosa (Reeve \& Carter, 1991).

Nas profundidades de 20 e $60 \mathrm{~cm}$, foram realizados experimentos de infiltração aplicando-se potenciais de $0,-3,-6$ e $-12 \mathrm{~cm}$ de água, com três repetições por potencial.

\section{RESULTADOS E DISCUSSÃO}

A estimativa dos pontos da curva de retenção da água no solo obtida pelos dois métodos, em campo e em laboratório, possibilitou a cobertura de uma boa gama de potenciais entre -1000 e 0 cm de água (Figura 1). A precisão das medidas na faixa de pressão comum aos dois métodos, $-300 \mathrm{a}-100 \mathrm{~cm}$, mostra a coerência dos dados experimentais. Observa-se menor variação da umidade volumétrica para a camada argilosa $(15-25 \mathrm{~cm}) \mathrm{em}$ relação à camada arenosa $(50-70 \mathrm{~cm})$ na faixa de pressões explorada.

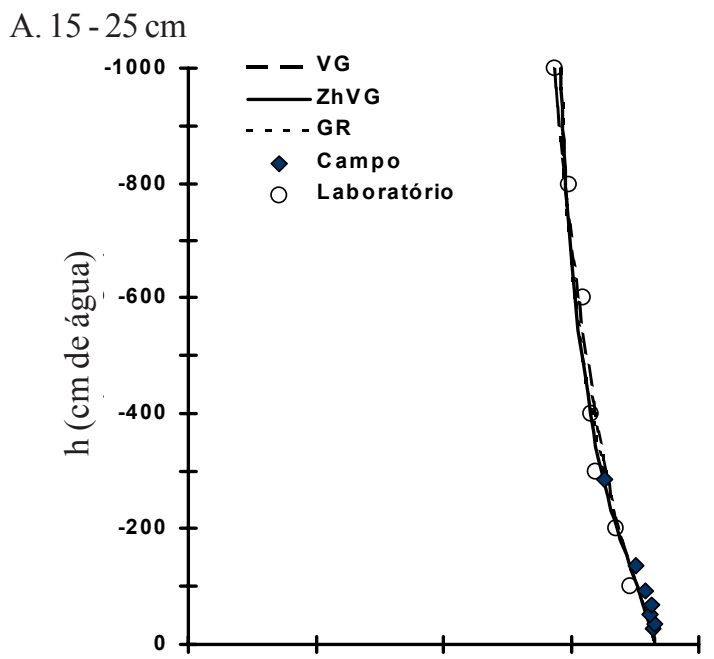

B. $50-70 \mathrm{~cm}$

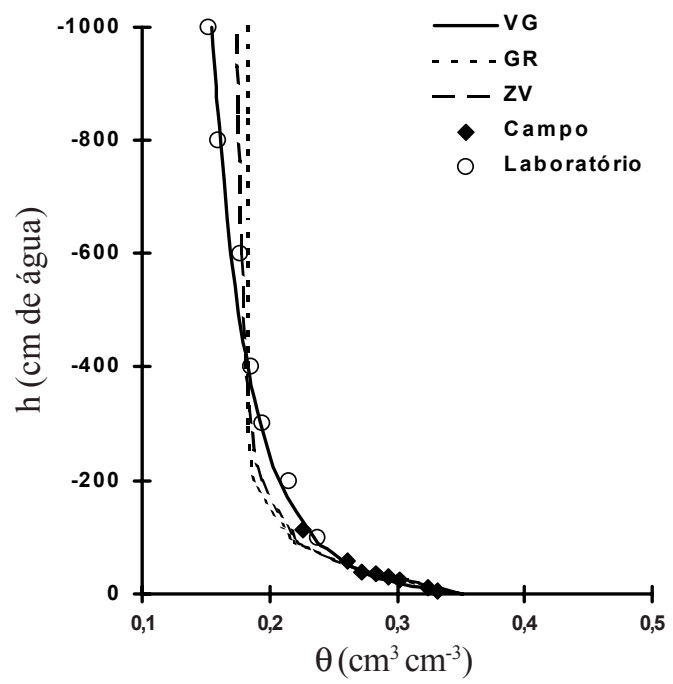

Figura 1. Curvas de retenção da água no solo, obtidas em campo e no laboratório, e ajustadas pelos funcionais propostos por: i) van Genuchten (1980) VG; ii) Russo (1988) GR; e iii) Zhang \& van Genuchten (1994) ZV, representativas das camadas de 15 - $25 \mathrm{~cm}(\mathrm{~A})$ e 50 - $70 \mathrm{~cm}$ (B)
Os modelos VG, GR e ZV, Eqs. 6, 11 e 15, foram ajustados, respectivamente, aos dados experimentais (Tabela 2). $\mathrm{O}$ valor de $\theta_{\mathrm{s}}$ é constante e medido experimentalmente em campo. Os parâmetros de ajuste são, portanto $\left(\theta_{\mathrm{r}}, \alpha\right.$ e n $)$, com o caso particular $\operatorname{de} \theta_{\mathrm{r}}=0$ para o modelo VG e os coeficientes de determinação, $\mathrm{R}^{2}$, indicam um bom ajuste dos diferentes modelos globalmente, embora na camada arenosa, $50-70 \mathrm{~cm}$, os modelos GR e ZV pareçam menos adequados que o modelo VG (Figura 1).

Tabela 2. Parâmetros dos funcionais propostos por: i) van Genuchten (1980), ii) Russo (1988) e iii) Zang e van Genuchten (1994)

\begin{tabular}{|c|c|c|c|c|c|c|}
\hline \multirow{2}{*}{$\begin{array}{c}\text { Camada } \\
\mathrm{cm}\end{array}$} & \multirow{2}{*}{ Modelos } & $\theta \mathrm{r}$ & $\theta \mathrm{s}$ & \multirow{2}{*}{$\begin{array}{c}\alpha \times 10^{-3} \\
\mathrm{~cm}^{-1}\end{array}$} & \multirow[b]{2}{*}{$\mathrm{n}$} & \multirow{2}{*}{$\mathrm{R}^{2}$} \\
\hline & & \multicolumn{2}{|c|}{$\mathrm{cm}^{3} \mathrm{~cm}^{-3}$} & & & \\
\hline \multirow{3}{*}{$15-25$} & VG & 0,000 & 0,466 & 4,12 & 1,117 & 0,965 \\
\hline & GR & 0,387 & 0,466 & 7,44 & 2,176 & 0,968 \\
\hline & ZV & 0,370 & 0,466 & 5,83 & 0,707 & 0,980 \\
\hline \multirow{3}{*}{$50-70$} & VG & 0,000 & 0,352 & 79,00 & 1,188 & 0,978 \\
\hline & GR & 0,182 & 0,352 & 39,13 & 1,497 & 0,958 \\
\hline & $\mathrm{ZV}$ & 0,169 & 0,352 & 20,11 & 1,955 & 0,976 \\
\hline
\end{tabular}

As diferenças na textura das duas camadas impõem comportamentos muito diferentes na infiltração (Figura 2) para as várias sucções aplicadas a cada uma das duas profundidades.

A. $15-25 \mathrm{~cm}$

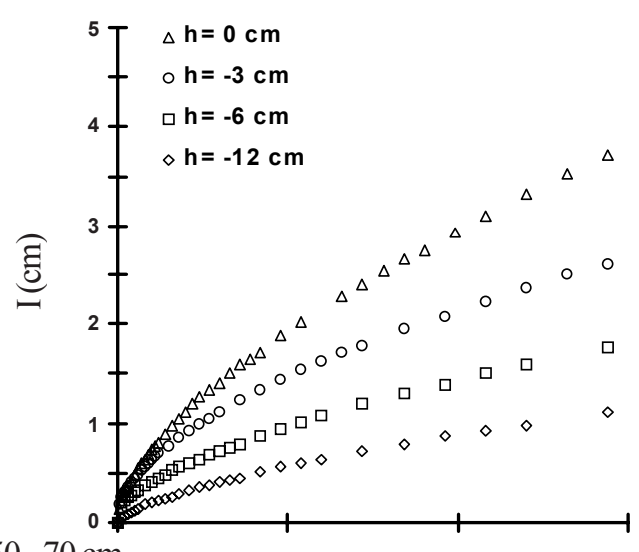

B. $50-70 \mathrm{~cm}$

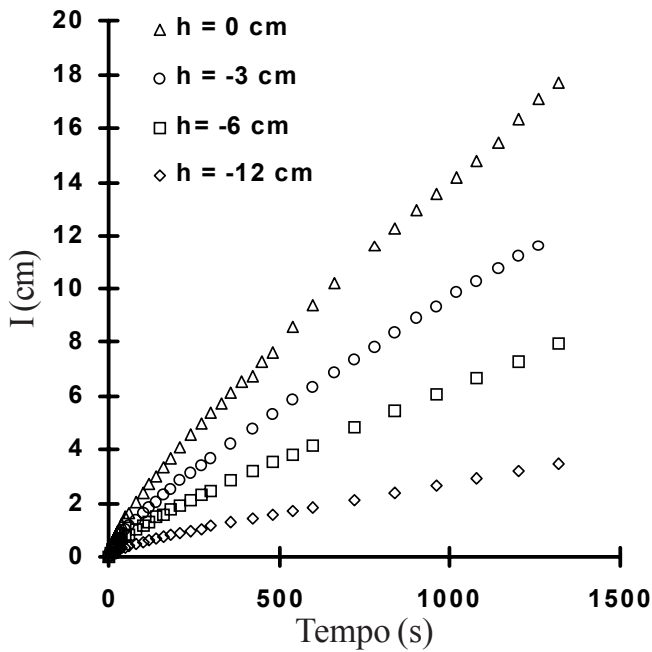

Figura 2. Infiltração acumulada (I) em função do tempo para os diferentes potenciais de fornecimento da água $(\mathrm{h})$ para as camadas argilosa, $15-25 \mathrm{~cm}(\mathrm{~A})$, e arenosa, $50-70 \mathrm{~cm}$ (B) 
Para o mesmo tempo de infiltração, as lâminas de água infiltradas são superiores no solo arenoso que no solo argiloso; esses valores diminuem com a redução da pressão; assim, na saturação $\left(\mathrm{h}_{0}=0\right)$ e após $1500 \mathrm{~s}$ de infiltração, a lâmina de água infiltrada foi de $3,7 \mathrm{~cm}$ para a camada argilosa, enquanto para a camada de areia foi de $19,6 \mathrm{~cm}$.

Todas as curvas de infiltração $\mathrm{I}(\mathrm{t})$ foram aproximadas pela Eq. 1, com coeficientes de determinação $\left(R^{2}\right)$ superiores a 0,995 (Tabela 3). As diferenças entre os valores de $C_{1}$ e $C_{2}$ de cada repetição foram devidas aos efeitos da variabilidade espacial, em pequena escala, das características da infiltração, dado que cada ensaio ocupa um local diferente, porém próximo. A Eq. 1 caracteriza o processo de infiltração a partir de um disco, devido às forças capilares e gravitacionais. $\mathrm{O}$ efeito dessas forças varia com o tempo de infiltração. No início do processo de infiltração, as forças gravitacionais podem ser desprezadas diante das forças capilares, e vice-versa; após um longo tempo de infiltração, as forças capilares podem ser desprezadas. A curvatura e a forma relativa das curvas de infiltração acumulada $\mathrm{I}(\mathrm{t})$, evoluem em função das características hidrodinâmicas, e estas curvas tendem a retas quando o fluxo de infiltração se aproxima do regime estacionário.

Tabela 3. Parâmetros $\mathrm{C}_{1}, \mathrm{C}_{2}$, e coeficiente de determinação $\left(\mathrm{R}^{2}\right)$, obtidos pelo ajuste da Eq. 1 aos dados da infiltração acumulada em função do tempo para os potenciais de $0,-3,-6$ e $-12 \mathrm{~cm}$, referentes às camadas de $15-25$ e 50-70 cm

\begin{tabular}{|c|c|c|c|c|c|}
\hline $\begin{array}{c}\text { Camada } \\
\mathrm{cm}\end{array}$ & Potenciais & Repetições & $\begin{array}{c}\mathrm{C}_{1} \\
\mathrm{~cm} \mathrm{~s}^{-1 / 2}\end{array}$ & $\begin{array}{c}\mathrm{C}_{2} \\
\mathrm{~cm} \mathrm{~s}^{-1}\end{array}$ & $\mathrm{R}^{2}$ \\
\hline \multirow{12}{*}{$15-25$} & \multirow{3}{*}{0} & R1 & 0,08253 & 0,00135 & 0,999 \\
\hline & & $\mathrm{R} 2$ & 0,13482 & 0,00133 & 0,999 \\
\hline & & R3 & 0,09277 & 0,00114 & 0,999 \\
\hline & \multirow{3}{*}{-3} & $\mathrm{R} 1$ & 0,05959 & 0,00061 & 0,999 \\
\hline & & $\mathrm{R} 2$ & 0,08561 & 0,00025 & 0,999 \\
\hline & & $\mathrm{R} 3$ & 0,13473 & 0,00034 & 0,997 \\
\hline & \multirow{4}{*}{-6} & $\mathrm{R} 1$ & 0,05385 & 0,00026 & 0,999 \\
\hline & & $\mathrm{R} 2$ & 0,04966 & 0,00004 & 0,998 \\
\hline & & R3 & 0,11378 & 0,00041 & 0,999 \\
\hline & & $\mathrm{R} 1$ & 0,05635 & 0,00008 & 0,999 \\
\hline & \multirow[t]{2}{*}{-12} & $\mathrm{R} 2$ & 0,01883 & 0,00038 & 0,997 \\
\hline & & $\mathrm{R} 3$ & 0,02530 & 0,00040 & 0,998 \\
\hline \multirow{12}{*}{$50-70$} & \multirow{3}{*}{0} & R1 & 0,15067 & 0,01173 & 0,999 \\
\hline & & $\mathrm{R} 2$ & 0,21234 & 0,01255 & 0,999 \\
\hline & & $\mathrm{R} 3$ & 0,27155 & 0,00725 & 0,996 \\
\hline & \multirow{3}{*}{-3} & $\mathrm{R} 1$ & 0,13656 & 0,00898 & 0,999 \\
\hline & & $\mathrm{R} 2$ & 0,11304 & 0,00647 & 0,999 \\
\hline & & R3 & 0,11822 & 0,00271 & 0,999 \\
\hline & \multirow{3}{*}{-6} & $\mathrm{R} 1$ & 0,12683 & 0,00863 & 0,999 \\
\hline & & $\mathrm{R} 2$ & 0,10447 & 0,00536 & 0,999 \\
\hline & & $\mathrm{R} 3$ & 0,09046 & 0,00543 & 0,999 \\
\hline & \multirow{3}{*}{-12} & $\mathrm{R} 1$ & 0,05271 & 0,00214 & 0,999 \\
\hline & & $\mathrm{R} 2$ & 0,03984 & 0,00191 & 0,999 \\
\hline & & R3 & 0,04735 & 0,00239 & 0,999 \\
\hline
\end{tabular}

Os valores de $\mathrm{C}_{1}$ são maiores que os de $\mathrm{C}_{2}$ (Tabela 3); conseqüentemente, vão contribuir também para aumentar $o$ intervalo de tempo no qual os efeitos capilares preponderam em relação aos efeitos gravitacionais. Para a camada argilosa, 15 - $25 \mathrm{~cm}$, a relação $\mathrm{C}_{1} / \mathrm{C}_{2}$ foi menor $\mathrm{em} \mathrm{h}_{0}=0$, sendo de 81 , que para as outras pressões $-3,-6,-12 \mathrm{~cm}$, sendo em média de 376 . Nesta camada existirá, portanto, uma perda de precisão na estimativa da condutividade hidráulica em relação à estimativa da sorvidade, quando a pressão aumentar o que pode ser atribuído ao fato de que os ensaios de infiltração foram paralisados antes que os efeitos gravitacionais fossem preponderantes em relação aos efeitos capilares. $\mathrm{O}$ menor valor da relação $\mathrm{C}_{1} / \mathrm{C}_{2}$ obtido em $\mathrm{h}_{0}=0$, mostra como, na saturação, os efeitos gravitacionais se manifestam com uma importância relativa maior, explicado pela presença de numerosas fissuras observadas e hidraulicamente ativas em $\mathrm{h}_{0}=0$, e não mais em $\mathrm{h}_{0}=-3 \mathrm{~cm}$, induzindo um escoamento mais rápido através desta macroporosidade.

Para a camada arenosa, 50 - $70 \mathrm{~cm}$, o valor da relação $\mathrm{C}_{1} / \mathrm{C}_{2}$ é de 22 em média para todas as pressões impostas, sem nenhuma tendência particular. Nesta camada, os efeitos gravitacionais são, portanto, importantes em relação aos efeitos capilares. Observa-se, neste caso, que o tempo dos ensaios de infiltração foi suficientemente longo para permitir uma boa estimativa de S e K.

O conhecimento das curvas de retenção $\theta(h)$ e dos valores de $\mathrm{C}_{1}$ e $\mathrm{C}_{2}$, para cada uma das repetições, possibilita calcular-se os fatores $A_{1}$ e $A_{2}$ pelos métodos $\mathrm{VG}, \mathrm{GR}$ e $\mathrm{ZV}$ e, em função do potencial imposto, os valores de $\mathrm{K}\left(\mathrm{h}_{0}\right)$ e $\mathrm{S}\left(\mathrm{h}_{0}\right)$ (Eqs. 4 e 5). A Figura 3 apresenta os valores médios da condutividade hidráulica e da sorvidade, em função da pressão imposta.

Os valores da condutividade hidráulica e da sorvidade, estimados pelos métodos VG, GR e ZV, foram maiores para a camada arenosa que para camada argilosa, estando em acordo com as diferenças observadas nas lâminas infiltradas.

$\mathrm{O}$ valor da condutividade hidráulica, estimada pelo método $\mathrm{VG}$, foi maior que a estimada pelos métodos GR e ZV, enquanto a sorvidade estimada é menor; assim, o método VG dá um peso mais importante à condutividade e, sobretudo, à saturação. $\mathrm{O}$ valor da condutividade, estimado pelo método $\mathrm{VG}$, é da ordem de 9 e 4 vezes para a camada argilosa, e da ordem de 20 e 7 vezes para a camada arenosa, maior que o valor da condutividade estimada pelos métodos GR e ZV, respectivamente. Em efeito, a curva de $\mathrm{K}(\mathrm{h})$ do modelo VG apresenta uma não linearidade mais forte que os modelos GR e ZV. Para esses dois métodos, a condutividade hidráulica varia com menor expressividade com a sucção. A forma da sorvidade com a sucção é semelhante para os três métodos, um comportamento linear entre as sucções $\mathrm{h}_{0}=-12,-6$ e $-3 \mathrm{~cm}$ e depois um aumento da inclinação até $\mathrm{h}_{0}=0$, particularmente brusco para o método $\mathrm{VG}$.

Os valores das condutividades hidráulicas normalizadas, $\mathrm{K} / \mathrm{K}_{\mathrm{s}}$, estimados pelos três métodos, foram comparados com os resultados teóricos fornecidos pelas Eqs. 7, 12 e 16 dos modelos $\mathrm{VG}, \mathrm{GR}$ e ZV, respectivamente (Figura 4). Os valores de $\mathrm{K}_{\mathrm{s}}$ foram aqueles estimados $\mathrm{a} \mathrm{h}_{0}=0$ para as duas profundidades.

Para a camada argilosa (15 - $25 \mathrm{~cm})$ (Figura 4A) o método VG possibilita uma estimativa mais próxima aos valores de $\mathrm{K}\left(\mathrm{h}_{0}\right)$ calculados pelo modelo teórico Eq. 7 correspondente, enquanto os métodos GR e ZV se distanciam de maneira importante dos seus respectivos modelos teóricos, Eqs. 12 e 16, fornecendo valores bem maiores que os estimados a partir dos dados de infiltração.

Resultados análogos foram observados para a camada arenosa (50-70 cm) (Figura 4B) em que o método VG fornece valores mais próximos dos valores teóricos. Os métodos GR e ZV apresentam um desvio relativo menos importante que para a camada argilosa, enquanto o modelo teórico ZV mostra valores 
A. $15-25 \mathrm{~cm}$

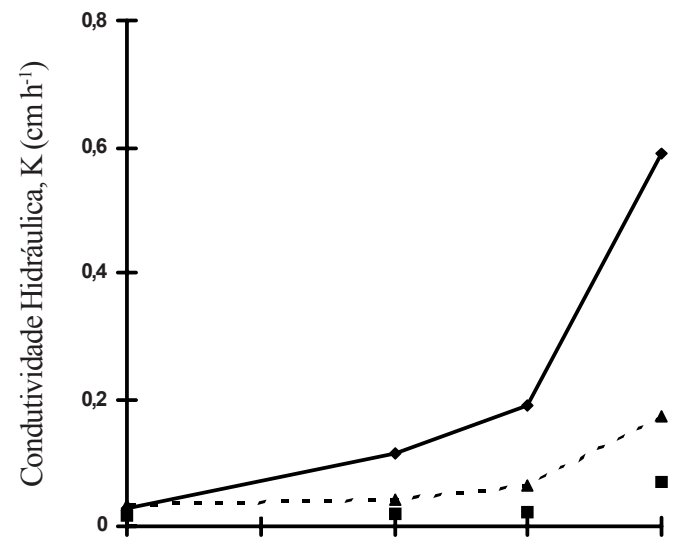

B. $50-70 \mathrm{~cm}$

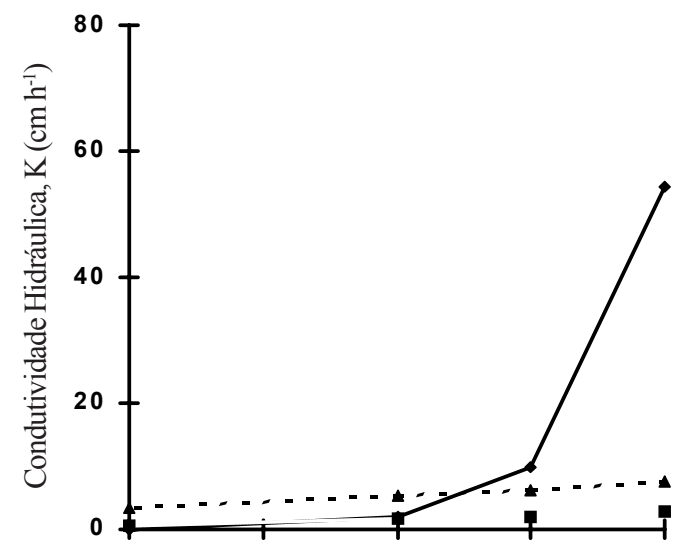

C. $15-25 \mathrm{~cm}$

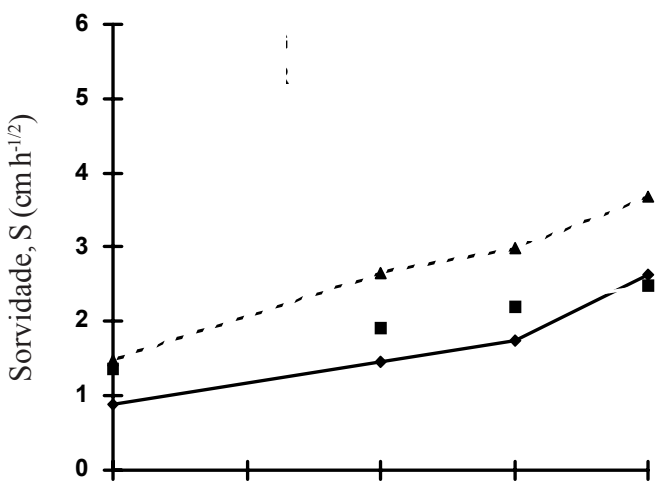

D. $50-70 \mathrm{~cm}$

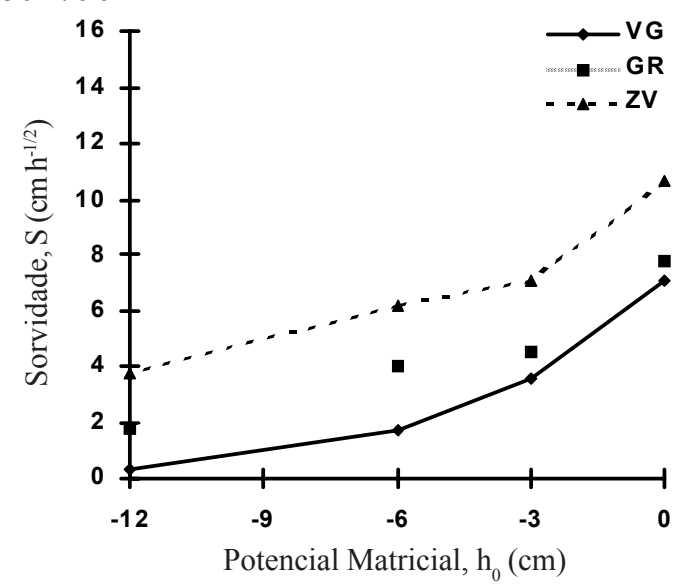

Figura 3. Condutividade hidráulica e sorvidade em função do potencial matricial, para as camadas argilosa $(15-25 \mathrm{~cm}) \mathrm{e}$ $\operatorname{arenosa}(50-70 \mathrm{~cm})$
A. $15-25 \mathrm{~cm}$

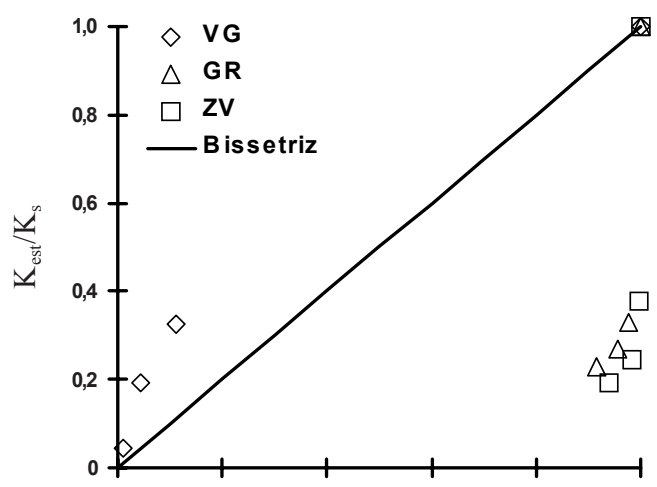

B. $50-70 \mathrm{~cm}$

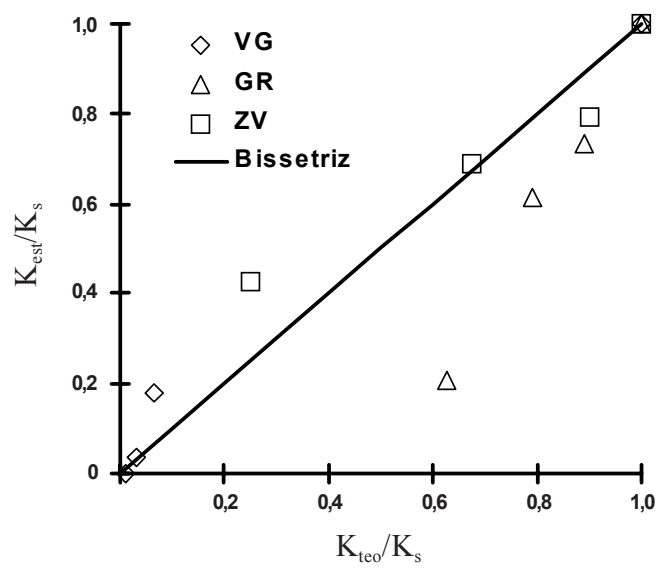

Figura 4. Condutividade hidráulica normalizada estimada em função da condutividade hidráulica normalizada teórica, para as camadas (A) argilosa $(15-25 \mathrm{~cm})$ e (B) arenosa $(50-70 \mathrm{~cm})$

que oscilam ao redor da bissetriz e os valores do modelo GR são sistematicamente superiores aos estimados.

Os modelos teóricos dependem dos valores de $\mathrm{K}_{\mathrm{s}}$ e dos parâmetros $\left(\alpha, \mathrm{n}, \theta_{\mathrm{s}}\right.$ e $\left.\theta_{\mathrm{r}}\right)$ da curva característica $\theta(\mathrm{h})$ enquanto os valores estimados pelos três métodos consideram a cinética da infiltração.

A análise estatística entre os valores das condutividades hidráulicas normalizadas $\mathrm{K} / \mathrm{K}_{\mathrm{s}}$ estimados pelos três métodos $\mathrm{VG}$, GR e ZV, e os valores calculados pelos modelos teóricos correspondentes, é apresentada na Tabela 4. O valor de EQM indica o grau de desvio entre as determinações experimentais e os valores calculados pelo modelo teórico correspondente, expresso como percentagem da média das determinações experimentais e o valor esperado de EQM tende para zero, quando os valores estimados e os teóricos tendem a ser iguais. Os menores valores de EQM (22,9 e 14,2\%) foram obtidos para o método VG para a camada argilosa, $15-25 \mathrm{~cm}$, e para o método ZV para arenosa, $50-70 \mathrm{~cm}$, respectivamente. O RD descreve a razão entre o espalhamento das determinações experimentais e o espalhamento dos valores calculados pelo modelo teórico correspondente. O EM indica se o modelo teórico fornece uma estimativa melhor das determinações experimentais que o valor médio dessas determinações. Em ambos os casos, o valor esperado tende a 1 (um), quando os valores estimados e aqueles do modelo teórico são consistentes. Valores de RD e EM próximo de um foram obtidos para o método VG, em ambas as camadas. O $\mathrm{CRM}$ indica se o modelo tende a superestimar $(\mathrm{CRM}<0)$ ou a subestimar $(\mathrm{CRM}>0)$; o valor esperado de $\mathrm{CMR}$ tende a zero, na 
ausência de desvios sistemáticos entre os valores teóricos e experimentais. Observa-se que o modelo GR superestima os valores para ambas as camadas. Globalmente, como mostra o resultado da análise estatística, o método VG parece ser o mais recomendado, pois o seu modelo teórico correspondente prediz razoavelmente bem a condutividade hidráulica normalizada para as duas camadas, enquanto o método GR parece ser o menos recomendado, pois o seu modelo teórico correspondente superestima sistematicamente e prediz mal a condutividade hidráulica normalizada, para ambas as camadas.

Tabela 4. Resultado da análise estatística* sobre a comparação entre os métodos e os modelos teóricos correspondentes, da condutividade hidráulica normalizada para as camadas de 15 - 25 e $50-70 \mathrm{~cm}$

\begin{tabular}{crrrr} 
Modelos & EQM & RD & EM & CMR \\
\hline \multicolumn{5}{c}{$15-25 \mathrm{~cm}$} \\
VG & 22,858 & 0,914 & 0,940 & 0,117 \\
GR & 128,522 & 0,388 & $-2,423$ & $-1,113$ \\
ZV & 134,903 & 0,375 & $-2,576$ & $-1,164$ \\
\hline \multicolumn{5}{c}{$50-70 \mathrm{~cm}$} \\
VG & 18,852 & 0,946 & 0,980 & 0,089 \\
GR & 37,664 & 1,519 & 0,296 & $-0,294$ \\
ZV & 14,191 & 0,511 & 0,750 & 0,029 \\
\hline
\end{tabular}

EQM: Erro quadrático médio; RD: Razão de desvios; EM: Eficiência da modelagem; CMR: Coeficiente de massa residual

\section{CONCLUSÕES}

1. A equação da infiltração acumulada em função do tempo, equação a dois termos, descreveu com precisão os ensaios de infiltração realizados, apresentando coeficientes de determinação superiores a 0,995.

2. Os valores da sorvidade e da condutividade hidráulica do solo, em condições de umidade próximo à saturação, estimados pelos métodos VG, GR e ZV, foram maiores para a camada arenosa que para a camada argilosa, devido às diferenças observadas nas lâminas de água infiltradas.

3. O valor da condutividade hidráulica na saturação, em $\mathrm{h}_{0}=0$, estimada pelo método VG, foi maior, da ordem de 9 e 4 vezes, para a camada argilosa, e da ordem de 20 e 7 vezes para a camada arenosa, que os valores estimados pelos métodos GR e ZV, respectivamente.

4. O método VG parece ser o mais recomendado na determinação da condutividade hidráulica e da sorvidade, pois o seu modelo teórico prediz com razoável precisão a condutividade hidráulica normalizada.

\section{LITERATURA CITADA}

Angulo-Jaramillo, R.; Gaudet, J.P.; Thony, J.L.; Vauclin, M. Measurement of hydraulic properties and mobile water content of a field soil. Soil Science Society America Journal, Madison, v.60, p.710-715, 1996.

Angulo-Jaramillo, R.; Vandervaere, J.P.; Roulier, S.; Thony, J.L.; Gaudet, J.P.; Vauclin, M. Field Measurement of soil hydraulic properties by disc and ring infiltrometers: A review and recent developments. Soil \& Tillage Research, Madison, v.55, p.1-29, 2000.
Borges, E.; Antonino, A.C.D.; Dall'Olio, A.; Audry, P.; Carneiro, C.J.G. Determinação das propriedades hidráulicas de solos não saturados utilizando-se permeâmetros a disco. Pesquisa Agropecuária Brasileira, Brasília, v.34, n.11, p.2083-2090, 1999.

EMBRAPA - Empresa Brasileira de Pesquisa Agropecuária. Centro Nacional de Pesquisa de Solos. Sistema brasileiro de classificação de solos. Brasília: EMBRAPA - Serviço de Produção da Informação; Rio de Janeiro, EMBRAPA solos, 1999. 412p.

Gardner, W.R. Some steady-state solutions of the unsaturated moisture flow equation with application to evaporation from a water table. Soil Science, Baltimore, v.85, p.228-232, 1958.

Haverkamp, R.; Ross, P.J.; Smettem, K.R.J.; Parlange, J.Y. Three dimensional analysis of infiltration from the disc infiltrometer. 2. Physically based infiltration equation. Water Resources Research, Washington, v.30, p.2931-2935, 1994.

Loague, K.M.; Green, R.E. Statistical and graphical methods for solute transport models. Journal of Contaminant Hydrology, Amsterdam, v.7, p.51-73, 1991.

Perroux, K.M.; White, I. Designs for disc permeameters. Soil Science Society America Journal, Madison, v.52, p.1205-1215, 1988.

Reeve, M.J.; Carter, A.D. Water release characteristic. In: Smith, K.A.; Mullins, C.E. (ed.). Soil analysis - Physical methods. New York: Marcel Dekker, Inc, 1991. cap. 3, p.111-160.

Russo, D. Determining soil hydraulic properties by parameter estimation on the selection of a model for hydraulic properties. Water Resources Research, Washington, v.24, p.453-459. 1988.

Smettem, K.R.J.; Clothier, B.E. Measuring unsaturated sorptivity and hydraulic conductivity using multi-disc permeameters. Journal of Soil Science, Oxford, v.40, p.563-568, 1989.

Soil Taxonomy. A basic system of soil classification for making and interpreting soil survey. United States Department of Agricultural - USDA. Natural Resources Conservation Service, Washington, DC. 1999. 870p.

Thony, J.L.; Vachaud, G.; Clothier, B.E.; Angulo-Jaramillo, R. Field measurements of the hydraulic properties of soil. Soil Technology, Cremlingen, v.4, p.111-123, 1991.

Vandervaere, J.P.; Peugeot, C.; Vauclin, M.; Angulo-Jaramillo, R.; Lebel, T. Estimating hydraulic conductivity of crusted soils using disc infiltrometers and minitensiometers. Journal of Hydrology, Amsterdam v.188-189, p.203-223, 1997.

van Genuchten, M.Th. A closed-form equation for predicting the hydraulic conductivity of unsaturated soils. Soil Science Society America Journal, Madison, v.44, p.892-898, 1980.

Warrick, A.W.; Broadbridge, P. Sorptivity and macroscopic capillary length relationships. Water Resources Research, Washington, v.28, p.427-431. 1992.

Zhang, R. Determination of soil sorptivity and hydraulic conductivity from the disc infiltrometer. Soil Science Society America Journal, Madison, v.61, p.1024-1030, 1997.

Zhang, R.; van Genuchten, M.Th. New models for unsaturated soil hydraulic properties. Soil Science, Baltimore, v.158, p.77-85, 1994. 